\title{
Use of information technology for auditing effective use of laboratory services
}

\author{
M Peters, P M G Broughton, P G Nightingale
}

\section{Introduction}

Medical audit has been defined as the systematic, critical analysis of the quality of medical care, including the procedures used for diagnosis and treatment, the use of resources, and the resulting outcome and quality of life for the patient. ${ }^{1}$ For pathology this implies the analysis of the quality of the pathology service in terms of its contribution to patient care. This process will involve both the clinician who uses diagnostic services and the laboratory staff who provide them.

To help in auditing pathology laboratories accreditation procedures are being developed which cover organisational and management aspects, such as staffing, equipment, test repertoire, workload, safety and technical performance. ${ }^{2}$ Most of these are inward-looking features concerned with the provision of an efficient service, however, and do not cover the use made of this service by the clinician-that is, the effectiveness, relevance, or appropriateness of investigations-or deal with the often repeated criticism that many laboratory tests are unnecessary and a waste of resources. ${ }^{3-5}$ Laboratories usually lack the information about outcome which is necessary to assess the clinical impact of their work, and ultimately the appropriateness of an investigation can only be judged by the clinician with responsibility for care of the patient. Quality assurance, as at present practised by laboratories, is mainly concerned with ensuring that the result is right, but it now needs to be broadened to ensure that the right test is done at the right time on the right patient. ${ }^{2}$

Assessment of these features requires quantitative data, often from different sources, and information technology will be essential for measuring, integrating, processing and presenting the findings. This paper describes some mechanisms for auditing the effective use of laboratory services and shows how this approach may be developed jointly by the clinician and the laboratory to improve the quality of laboratory services and their contribution to patient care.

Wolfson Research

Laboratories, Queen

Elizabeth Medical

Centre, Birmingham

B15 2TH

$M$ Peters

P M G Broughton

P G Nightingale

Correspondence to:

Margaret Peters

Accepted for publication

20 March 1991

\section{Measuring quality}

Various external quality assessment (EQA) schemes for pathology now provide data about some aspects of performance which can be used in comparing different laboratories and for assessing changes with time. This form of peer review has been widely accepted by the profession and, in the absence of other standards of accuracy, the consensus value is now generally regarded as the desirable goal. A similar approach could be adopted in clinical audit. Here the main need is for quantitative data, not only on diagnostic accuracy and technical performance, but also on the contribution of laboratory work to standards of patient care, including factors such as timeliness and the appropriate use of investigations. ${ }^{1}$

\section{Measuring demand}

Laboratory workload data provide a simple form of audit, and show wide differences in the numbers and types of tests done on similar patients in different countries, in different hospitals within a country, and by different clinicians within a hospital (fig 1). Many reasons for these variations have been suggested and strategies for improvement proposed. ${ }^{6}$ Variations are largest between countries, (such as North America and the United Kingdom, and are smallest within a hospital, which is where clinical audit starts. Although it is usually the clinician who is blamed for unnecessary testing, sometimes the laboratory either selects the tests to be done in response to a request-for example, for thyroid function testing-or does different or additional tests. $^{7}$

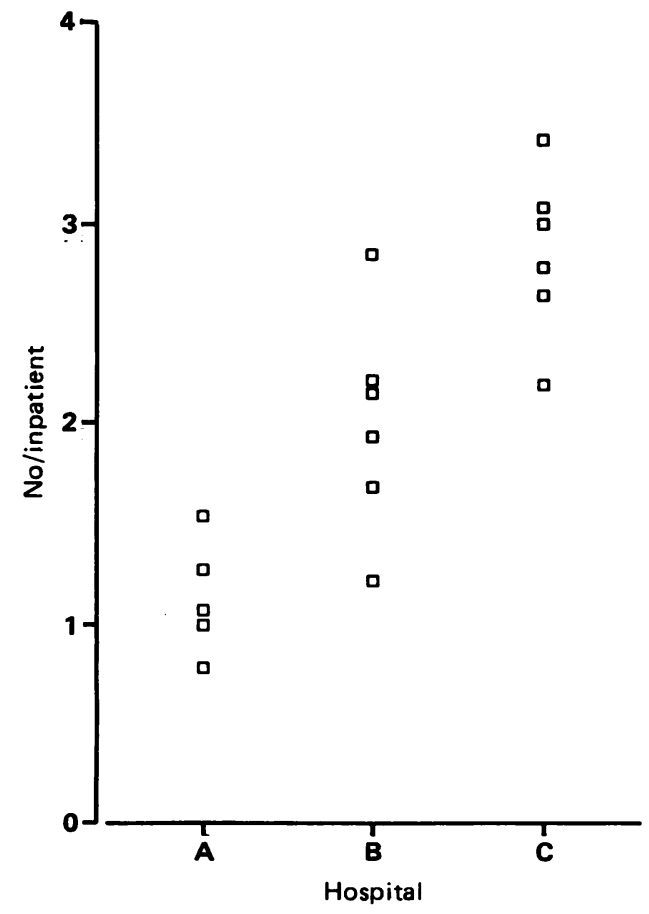

Figure 1 Average number of plasma electrolyte measurements per inpatient admission made in one month by general physicians at three hospitals (each point represents one physician). 
Although unnecessary tests undoubtedly occur, they are almost impossible to define because of the varying importance which clinicians place on laboratory investigations: a test which one regards as essential may, in a similar clinical situation, be regarded as less important or unnecessary by others. Although laboratory overuse has attracted most attention, underuse and misuse ${ }^{8}$ also occur, but are more difficult to identify and quantify. There is little evidence, however, that any of these differences affect patient care, but this may change when it becomes possible to correlate data on laboratory use (which are, at best, on one computer) with measures of clinical outcome (which are on another).

The appropriateness or effectiveness of an investigation depends on whether it influences the clinical decision, but in practice this can only be assessed from some measure of outcome, such as morbidity, mortality, bed stay, number of admissions or operations and (for general practitioners) referral rates.

The first step in this process is to inform clinicians of what tests they do and how much these cost. This immediately highlights variations between and within specialties (fig 2), and identifies those users and tests which consume most laboratory resources: attention can then be focussed on these. Simple computer packages for this are available. Ideally data from all service departments, including radiology, should be presented at the same time, thereby giving the clinician a comprehensive picture of all the investigations done.

The data can be presented numerically or as graphs, histograms, etc, so that clinicians can compare their usage and expenditure over a period of time and with their peers. These data soon lose their impact, even when expressed in terms of individual patients, because they are not related to clinical activity or outcome. In the example shown in fig 3 there were wide differences in the number of tests done annually by five general surgeons. These differences were reduced when the data were expressed in terms of numbers of patients admitted. When case mix was taken into account, there was a four-fold difference in tests done in cases of inguinal hernia, which

Figure 2 Clinical chemistry use and expenditure for medical specialities in one hospital in 1987-8.

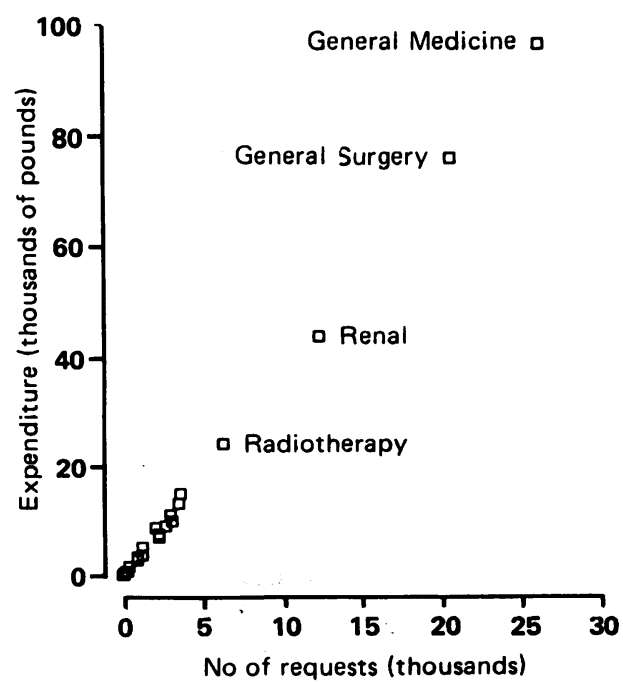

was reduced but not eliminated when only uncomplicated cases were considered. These results suggest that measures of clinicians' use of the laboratory should take account of the numbers and types of patients seen: simple comparisons of usage can be misleading. This type of data is obviously invaluable in audit, but the key question is whether it changes behaviour.

\section{Feedback}

Simple feedback of information about the numbers of tests requested and their costs has had variable success in persuading clinicians to modify their test requesting patterns. ${ }^{46}$ Factors which influence the success of feedback $^{910}$ are as follows: the attitudes of senior staff; the involvement of junior house staff who make the most requests; prompt and clear presentation of data; and the use of guidelines, fact sheets, seminars, etc.

The development of a consensus approach to test requesting, which is implicit in the use of feedback, has several potential advantages: ${ }^{9}$ (1) it provides a framework for constructive discussion between clinical and laboratory staff; (2) it makes no assumption about which tests are or are not necessary; (3) it is free from externally imposed standards and therefore non-threatening to the clinician; (4) it recognises that clinical practice is dynamic with no explicit ideal; (5) it can be automated by the use of information technology.

\section{Protocols}

Individual laboratories and clinicians both use protocols to define what they should do in certain circumstances. These protocols are often incomplete, informal, and unwritten and reflect the experience and wishes of senior staff. Variations in laboratory use by clinicians and in the ways laboratories respond to requests are due to differences between protocols and to difficulties in their routine application. The feedback process provides an opportunity to define protocols which represent consensus views. These can then be implemented through computerised decision support systems in which expertise is expressed in the form of rules. Although some decision-supported requesting systems have been developed, they have enjoyed variable success. ${ }^{41}$ Recent studies, however, indicate that decision supported request entry systems can be helpful both to clinicians and laboratories if they are integrated with hospital and laboratory systems. ${ }^{12}$ They can also provide automatic reinforcement of locally agreed protocols and prompt rather than dictate.

Decision-supported request entry systems have the following objectives:

(1) to facilitate the logical and predefined investigation of patients, and reduce variations occasioned by changes in junior medical staff;

(2) to save medical staff time through automatic generation of specimen labels and request documentation; 
Figure 3 Numbers of clinical chemistry tests done on inpatients by five general surgeons in one hospital in 1987-88. A) Total; B) per inpatient admission; $C$ ) per inguinal hernia admission; D) per uncomplicated inguinal hernia admission.
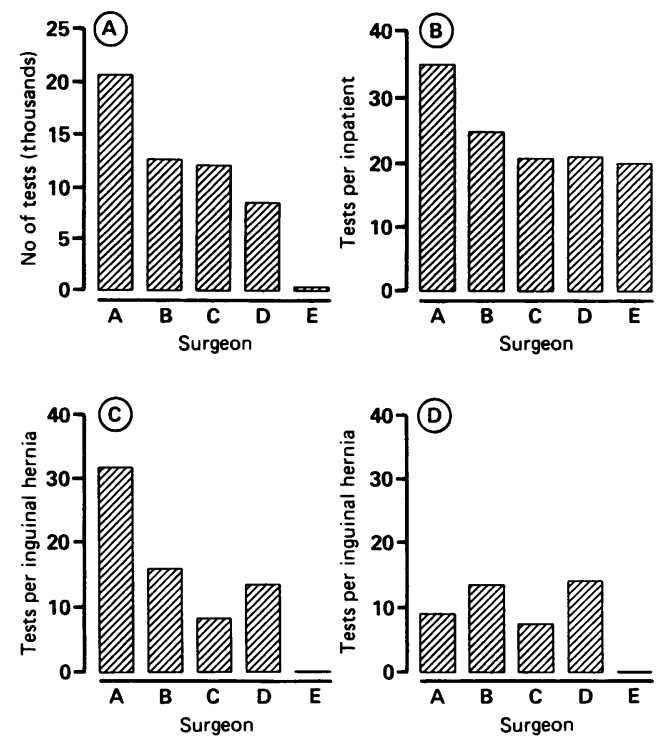

(3) to enable laboratories to know what investigations to expect, thereby providing them with an opportunity to manage demand for their services and to plan use of staff time and resources;

(4) to facilitate regular feedback to and discussion with users of the laboratory about optimal use;

(5) to facilitate postgraduate education and training of junior staff through the regular presentation of preferred practice.

The formulation of investigation protocols requires close collaboration between laboratory and clinical staff and some compromise between what is possible and what is desirable. After they have been introduced the effect of protocols on clinical practice and on laboratory use needs to be monitored closely and procedures refined in the light of experience.

The successful introduction of such systems requires that results and patient details are automatically extracted from laboratory and patient administration systems; that the protocols can be readily updated; that investigation schedules reflect current clinical state; and that exclusions and exceptions are allowed in atypical cases. Their use is encouraged by showing increased efficiency and savings in medical and laboratory staff time.

Decision supported reinforcement of investigation protocols ${ }^{5}$ may be either reactive or proactive. The former provides the requester with alarms, alerts, messages and reminders based on the nature of the request and the patient's previous investigations, and can be used for dealing with misuse, overrequesting, repeats and triggering of supplementary tests. Proactive systems provide, at ward level, specific investigation schedules for each patient and are suitable for specialist units where protocols are often already defined but difficult to implement manually. ${ }^{12}$ Both types of system can be implemented on inexpensive personal computers.

It will be important to assess the benefit of protocols to patient care. The development of local protocols will inevitably produce numerous variants for the same patient management conditions. Comparison of these protocols at a local, regional, or national level, especially where they can be related to outcome data, will produce much useful information but will not necessarily lead to the rapid development of national standards.

\section{Timeliness}

The timeliness with which laboratory reports are received by the clinician can affect patient care and the use of resources. ${ }^{15}$ Although published data are scanty, there are suggestions that turnaround times are seldom as good as the laboratory believes, and for general practitioners the situation is probably worse (fig 4). Sometimes the cause of the delay is not within the laboratory-for example, it may be in the collection and transport of the specimen or the delivery of the report to the right place. ${ }^{5}$ Responsibility for these delays needs to be clarified, and because they adversely affect the quality of service, the laboratory must ensure that appropriate action is taken. The first essential step towards improvement is for every laboratory to obtain more data by regularly monitoring the time between initiation of the request and receipt of the report. Only then will it be possible to assess whether this meets the clinician's requirements. ${ }^{13}$

The suggestion that clinicians should specify a "required by" time needs to be explored jointly by clinical and laboratory staff. There are obvious practical difficulties on both sides, and requirements will vary widely, but it would provide an incentive for laboratories to use their data management systems to schedule their work so that it more closely meets the needs specified by clinicians.

\section{Costs}

In the final analysis costs must be included in the assessment of quality ${ }^{5}$ and are in any case essential for laboratory management. ${ }^{14}$ Costs can be measured, although standard methods of doing this are still lacking. Administrators may demand them, but so far at least clinicians place more reliance on other aspects of quality. It seems inevitable that when all these other aspects of quality are equal (or hopefully equally good) then costs will enter into the auditing process, particularly when this involves comparison of the cost effectiveness of competing services.

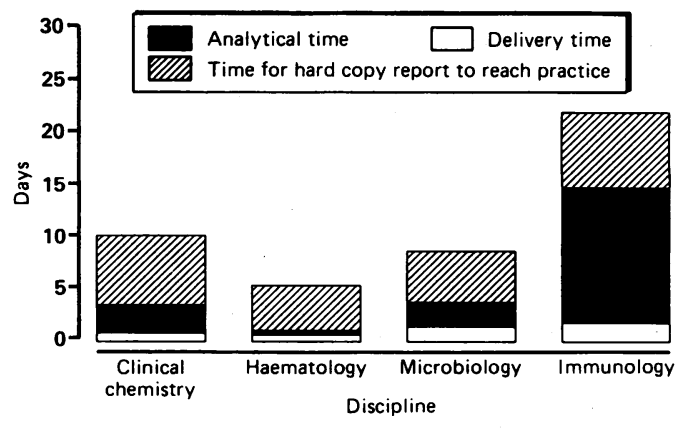

Figure 4 Average times taken for requested results to be returned to one general practice over three months. 


\section{What do you need?}

Ideally modern laboratory data management systems linked with satellite clinical decision support systems and Hospital Information Systems (HIS), providing ready access to a standardised core of clinical and operational data, are required if all the local audit measures described here are to be implemented systematically and on any appreciable scale. HIS are not yet generally available, however, and fully integrated decision supported requesting of laboratory investigations is at an early stage of development in this country.

Audit of effectiveness requires both clinically related data and laboratory operational data detailing sources and workload. These data are currently available to many laboratories, as is the ability to mix them. Although there are still some technical and operational issues to be addressed this mixing process will become easier when case mix systems are installed. Some of the required clinical data are already available in computer readable form from District Information Systems.

Current or local deficiencies in information technology infrastructures should not inhibit local audit initiatives, and modest studies may be carried out using ordinary personal computers using manually gathered operational data and commercially available statistical, graphical, and spreadsheet packages, without needing to write programs. Such studies should help to clarify objectives, identify problems, and provide insight into data processing techniques. Motivation, interest, numeracy and some understanding of information technology are essential, and it is difficult to overstate the importance of computer literacy in implementing the management techniques called for in future laboratory services.

\section{Conclusions}

The approach to audit which is outlined here involves four stages:

(1) measuring one or more aspects of laboratory utilisation such as numbers of tests, expenditure, timeliness of reports etc, and expressing the data in terms of clinical activity and some measure of patient outcome;

(2) comparing data among users of the laboratory;

(3) using feedback to develop a consensus which can be embodied into a protocol which is both practical and acceptable to the majority;

(4) implementing this by developing computer based decision support systems, and monitoring the data to see whether differences have narrowed and effectiveness improved.

This approach meets several of the recommendations made in the recent Audit Commission Report ${ }^{5}$ - namely, that pathology laboratories should measure and cost their workloads, collaborate with clinicians in developing protocols for requesting tests, and evaluate the response times needed for different types of test. Like EQA, the audit process is intended to do more than passively monitor the current situation. It depends on quantifiable rather than subjective observations, and uses information technology to inform and educate participants and encourage a consensus. For some specialties this can start at a local level, but for others it may need to be more widely based to obtain sufficient data to make the comparisons which are the main stimulus to improvement. Movement towards a consensus will usually mean that some use will increase and some decrease. Overall expenditure may therefore not change, but resources should be used more effectively.

As in the early days of EQA, auditing techniques now need to be developed through multicentre experimental studies involving both clinicians and laboratory staff. The main objectives of these will be to investigate various measures of laboratory effectiveness within each pathology discipline and for different clinical specialties, and to evaluate ways in which this information can be applied to improving patient care and the use of resources.

We thank the many colleagues who supplied data used in these studies and Dr James Neuberger and Dr Rousseau Gama for helpful discussions. Acknowledgement is made of financial support by the Department of Health

1 Department of Health. The Quality of Medical Care. Report of the Standing Medical Advisory Committee for the Secretaries of State for Health for England and Wales. Lecretaries of State for

2 Williams ED, Coleman JC, Emerson PM, Pennington GW, Lilleyman JS. Royal College of Pathologists' United Kingdom pilot study of laboratory accreditation. J Clin Pathol 1990;43:89-81.

3 Griner PF, Glaser RJ. Misuse of laboratory tests and diagnostic procedures. $N$ Engl J Med 1982;307:1336-9.

4 Young DW. Improving laboratory usage: a review. Postgrad Med J 1988;64:283-9.

5 Department of Health. The Pathology Services. A Management Review. The Audit Commission for Local Authorities and the National Health Service in England and Wales. London: HMSO, 1990.

6 Fraser CG, Woodford FP. Strategies to modify the test requesting patterns of clinicians. Ann Clin Biochem 1987;24:223-31.

7 Broughton PMG, Worthington DJ. Laboratories respond differently to the same clinical request. Ann Clin Biochem 1989;26:119-21.

8 Gibb I, Campbell Cowan J, Parnham AJ, Thomas TH. Use and misuse of a digoxin assay service. $\mathrm{Br}$ Med $J$ 1986; 293:678-80.

9 Gama R, Nightingale PG, Broughton PMG, et al. Feedback of laboratory usage and cost data to clinicians: does it alter requesting behaviour? Ann Clin Biochem 1991;28:143-9.

10 Bareford D, Hayling A. Inappropriate use of laboratory services: long term combined approach to modify request patterns. Br Med J 1990;301:1305-7.

11 Connelly DP. Embedding expert systems in laboratory information systems. Am J Clin Pathol 1990;94 (suppl 1):S7-S14.

12 Peters M, Clark IR, Parekh J, et al. Automatic application of rule-based decision support: a specialist unit investigation manager. Current pers British Journal of Healthcare Computing 1991:129-36.

13 Hilborne LH, Oye RK, McArdle JE, et al. Use of specimen turnaround time as a component of laboratory quality. $\mathrm{Am}$ turnaround time as a component

14 Broughton PMG, Woodford FP. Benefits of costing in the clinical laboratory. J Clin Pathol 1983;36:1028-35. 